EPJ Web of Conferences 103, 04012 (2015)

DOI: $10.1051 /$ epjconf/ 201510304012

(C) Owned by the authors, published by EDP Sciences, 2015

\title{
Questions and Answers in Behaviour of EBCD Carbon Monoxide Laser Radiation Pulses
}

\author{
T.E. Ventslavovich a and V.S. Kazakevich \\ P.N. Lebedev Physical Institute Samara Branch, Novo-Sadovaya St., 221, Samara, Russia
}

\begin{abstract}
In the work, the empirical Hurst's law, the wavelet-analysis and mathematical methods of nonlinear dynamics are used for the first time for the EBCD laser output pulses analysis. It has been shown that among a great number of the processes occurring in active medium, there is certain process manifests itself in peculiar features of the laser radiation time characteristics and introduces an element of unpredictability. The criterion is offered for the correctness of EBCD laser mathematical models being developed. The research of the frequency structure of EBCD laser output pulses was carried out on the base wavelet-analysis. It is shown that the transition to chaos in the EBCD laser dynamic system occurs through the period duplication..
\end{abstract}

Keywords: nonlinear effects, Hurst's law, an element of unpredictability, the period duplication.

In this work, the empirical Hurst's law, the wavelet-analysis and mathematical methods of nonlinear dynamics are used for the first time for the EBCD laser [1] output pulses analysis.

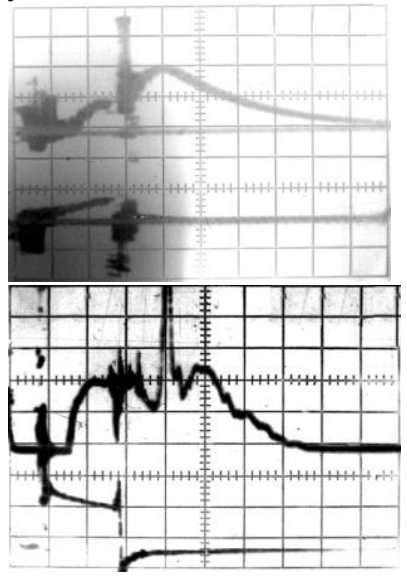

mix CO:N2 =1:9
Non-selective
resonator
$7 \rightarrow 6(\mathrm{P}=16)$
Qvkl.=193.7
$\mathrm{J} / \mathrm{L} \times$ Amaga
$\lambda=1923.43 \mathrm{~cm}^{-1}$
mix CO:N2 :He
$=1: 9: 10$
Non-selective
resonator
$6 \rightarrow 5(P=17)$
Qvkl. $=$
$J / L \times A m a g a$
$\lambda=1932.04 \mathrm{~cm}^{-1}$

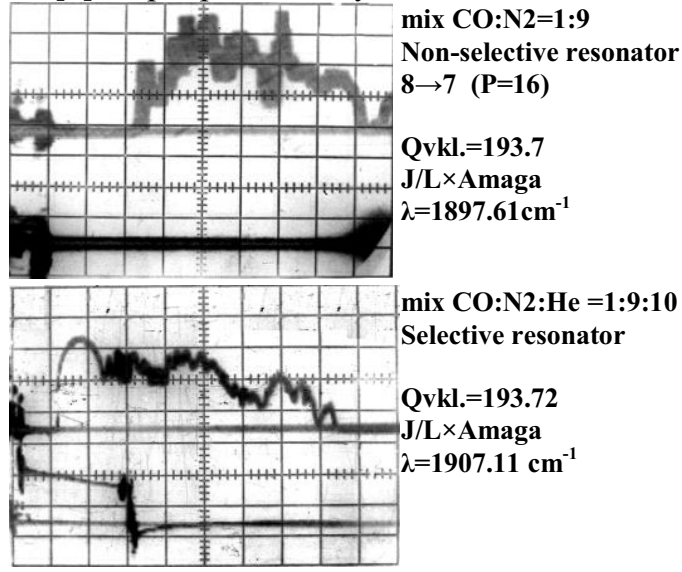

Figure 1. The output pulses of $\mathrm{EBCD}$ laser for separate vibration-rotation transitions for various resonators and types of mixes, $\mathrm{N}=0.5$ Amaga.

\footnotetext{
${ }^{\mathrm{a}}$ Corresponding author: venclavovich@gmail.com
}

This is an Open Access article distributed under the terms of the Creative Commons Attribution License 4.0, which permits unrestricted use, distribution, and reproduction in any medium, provided the original work is properly cited. 
It has been shown, that the received values of the Hurst parameter [2] within a wide spectral range don't depend on the specific power contribution and for the gas mix CO:N2 $=1: 9$ this parameter is equal to $\mathrm{H} \sim 0,84$. For the mix $\mathrm{CO}: \mathrm{N} 2: \mathrm{He}=1: 9: 10$ this value is $\mathrm{H} \sim 0,87$.

It is concluded, that time numbers of the EBCD laser radiation pulses are persistent and they are fractals [3].

As $\mathrm{H}<1$, it means that among a great number of processes occurring in the active medium there is certain process manifests itself in peculiar features of the laser radiation time characteristics and introduces an element of unpredictability.

The following criterion is offered for the correctness of EBCD laser mathematical models being developed: the value of Hurst parameter of the output pulses time numbers calculated theoretically should correspond to the parameter experimentally obtained.

In the work it is shown that the overall performance of the system is extreme at the border of the Hurst parameter change. So it is reasonable to generalize that this conclusion can be referred to the functioning of any difficult dynamic system.

The research of the frequency structure of the EBCD laser radiation pulses was carried out on the base wavelet-analysis [4]. For the first time the wavelet - spectra analysis of the EBCD laser output pulses of allowed to reveal the dependence $\mathrm{a}_{2} / \mathrm{a}_{1} \approx 2$, where $a_{1}$ and $a_{2}$ are components of the two time scales on the of analyzed signals wavelet -spectrum.

The detailed analysis of the kinetic equations allowed finding the reason for the given dependence. The equation of the Feigenbaum [5] type, $\alpha_{i+1}=\left(1+\lambda_{1}\right) \alpha_{i}-\lambda_{2} \alpha_{i}^{2}$ describing the behavior of the weak signal strengthening factor was obtained.

The analysis of the given equation properties showed, that in case of the EBCD laser pulse rating the time path for radiation pulse show the transition to the determined chaos if the time of the threshold gain doubled value achievement for separate vibration-rotation transitions of a $\mathrm{CO}$ molecule doesn't exceed the characteristic time of the vibration - vibration exchange. The required condition is quite realizable for the EBCD laser.

Thus, the transition to chaos in the EBCD laser dynamic system occurs through the period duplication.

\section{References}

1. V.S. Kazakevich Spectral-temporal characteristics of EBCD laser output pulses: dissertation, (Moscow, FIAN, 1985 (in Russian))

2. T. E. Ventslavovich, V. S. Kazakevich The R/S -analysis of radiation time series of pulse EBCD CO-laser, News of the Samara center of science of the Russian Academy of Sciences, 9, №3, , p.610-614. (2007) (in Russian)

3. A.E. Serikov, Fractal analysis of time numbers, Sociology 4M.2006.3 №22 (in Russian)

4. N.M. Astafieva, Uspekhi Fiz. Nauk 166, №1 p.1145-1170 (1996) (in Russian)

5. M. Feigenbaum, Uspekhi Fiz. Nauk 141 № 2. p. 343-374 (1983) (in Russian) 